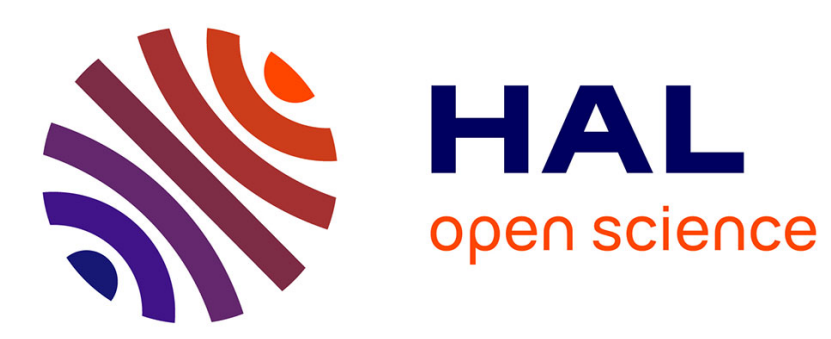

\title{
Control of a Silicone soft tripod robot via uncertainty compensation
}

\author{
Gang Zheng
}

\section{To cite this version:}

Gang Zheng. Control of a Silicone soft tripod robot via uncertainty compensation. IEEE Robotics and Automation Letters, 2020, 5 (2), pp.2801 - 2807. 10.1109/LRA.2020.2974714 . hal-02487883

\section{HAL Id: hal-02487883 \\ https://hal.inria.fr/hal-02487883}

Submitted on 21 Feb 2020

HAL is a multi-disciplinary open access archive for the deposit and dissemination of scientific research documents, whether they are published or not. The documents may come from teaching and research institutions in France or abroad, or from public or private research centers.
L'archive ouverte pluridisciplinaire HAL, est destinée au dépôt et à la diffusion de documents scientifiques de niveau recherche, publiés ou non, émanant des établissements d'enseignement et de recherche français ou étrangers, des laboratoires publics ou privés. 


\title{
Control of a Silicone soft tripod robot via uncertainty compensation
}

\author{
Gang Zheng
}

\begin{abstract}
Soft robot is an emergent research field which has variant promising applications, and the control of such robots is still challenging. Unlike using different techniques (such as Beam theory, Cosserat theory or high dimensional finiteelement method) to model the dynamics of soft robots, this paper introduces a simplified nominal model with uncertainty to describe its dynamic behavior. The link between this simple model and the finite-element method has been established, and a robust controller is proposed, by compensating the uncertainty which is estimated in a finite time by applying different types of estimators. The experiments have been made for different scenarios, and the corresponding results show the efficiency of the proposed method.
\end{abstract}

\section{INTRODUCTION}

Soft robot is an emergent research topic in robotics. In the literature, different definitions of soft robot can be found, ranging from soft actuators plus rigid body to soft material body with rigid or soft actuators [1]. Note that one of the key ideas of designing soft robot is to use deformable material to increase its reachability or to provide safe contact, therefore we roughly classify soft robot into two categories: one is for the rigid body with soft actuators, and the second one refers to the deformable body due to the flexibility of materials. The first category can be seen as a natural extension of traditional rigid robot, while the second one is totally new, and more attractive since it provides the flexibility to robots, for example, to adjust their shapes to suit the task and their environments. Due to the soft property, this type of robot can easily achieve compliant and safe tasks.

For the first type of soft robot, the controller design problem is relatively less complicated, since we can adapt traditional methods by taking into account the flexible dynamics introduced by soft joints or actuators. For example, the control of an anthropomimetic robot has been investigated in [2], which was based on the modeling of rigid body, and the classical computed-torque method has been applied to design the controller. Model predictive control (MPC) approach has also been used to control an inflatable humanoid robot in [3], which was based on the optimization of a given cost function under certain constraints, including dynamical constraints governed by the nonlinear dynamics. It is clear that the MPC method heavily relies on the precise model derived from the investigated robots.

The author is with Inria Lille, Villeneuve d'Ascq 59650, France, This work was supported in part by the Region Hauts-de-France, in part by the Project Inventor (I-SITE ULNE, le programme d'Investissements d'Avenir, Métropole Européenne de Lille), and in part by the Project ROBOCOP [ANR PRCE 19 CE19].
For the soft robots with deformable body, i.e., the second category, it is still a difficult problem when considering the control of such soft robots. The control theory developed for rigid robot is poorly applicable in this case [4]. It is mainly due to the lack of efficient method to obtain its exact model (either kinematic, or dynamic). Consequently, two different methodologies can be found in the literature, with or without the knowledge of the model, to control soft robots. Without the knowledge of the model, classical PID control was applied, which however cannot provide sufficient performance in practice (such as precision, rapidity, robustness etc.), since there does not exist a constructive way to tune the parameters of PID controller for multiple coupled inputs system.

Besides, other researchers tried to obtain the model of soft robots, and then design the corresponding controller, i.e., model-based controller design scheme. For this, different techniques were applied to seek the model of soft robot. One of the most used techniques is based on the curvature information of soft robot. The kinematic model was obtained for a hyperredundant robot by using the information of backbone curves in [5]. The kinematic model is obtained by using geometric information, and then a computed-torque controller is applied to control eel-like soft robot in [6]. Generally, the kinematic model of continuum soft robots was obtained by assuming that its curvature is piece-wise constant [7]. Based on this assumption, a robust feedback control was proposed in [8] to control the trajectory of soft robot. Other techniques have also been used to deduce the model of soft robot. For example, Euler-Bernoulli beam theory was used to model an inflatable robot in [9], and a force based feedback controller has been designed to control such a soft robot. Cosserat rod theory has been used to obtain a static model of a special continuum soft robot in [10], and a 3D steady-state model of a tendon-driven continuum soft octopus-like manipulator has been developed in [11]. Note that the curvature-based technique implicitly requires that the body shape of the soft robot should be in some sense uniform. This requirement is for the purpose of the model simplification, otherwise cumbersome computations need to be effectuated by dividing the whole non-uniform body into small pieces of uniform parts. Such an idea is in fact equivalent to a concept, called FEM (finite-element method), which is well known in the mechanic community. Via spatial discretization, FEM is used to obtain an approximated model for soft robots. Based on FEM model, a position feedback controller has been realized in [12] to regulate the position of a soft silicone robot, by solving a quadratic programming problem. However, it is well known that tiny mesh needs to be used in order to obtain an accurate approximated model, 
and this leads to high dimensional FEM model. Therefore, controller based on this approach is time-consuming.

Another technique used to obtain the model of soft robot is based on machine learning. In [13], the machine learning algorithm was used to compensate the dynamic uncertainties to control continuum robots. The forward dynamic model of a soft elastomer manipulator has been learned via a class of recurrent neural network in [14], based on which a locally optimal open-loop controller has been designed. In [15], a task space dynamic controller has been proposed for a soft robotic manipulator, which is based on the learned dynamic model. In [16], a neural network was used to learn the inputoutput model of a soft silicone robot, and robust controllers have been proposed to achieve the control tasks. For those mentioned methods based on machine learning technique, the main disadvantage is that the training phase for soft robots needs to collect enough data for the purpose of largely covering the robot's workspace. Moreover, the learned model does not take into account the external disturbance. Those disadvantages limit the application scenarios of such a method.

In this paper, we investigate the controller design problem for a specific portable soft material $\operatorname{robot}^{1}$ (see Fig. 1), which is originally designed for education/tutorial purpose. Such a robot can be regarded as a soft version between the cable robots and the rigid parallel robot for the pick-and-place application via soft links, in order to provide safe contact with its surrounding environment. In our work, we will not struggle to obtain a precise model for the investigated soft robot, but propose a simplified nominal model with uncertainty, where the uncertain term represents the mismatched error between the exact model and the nominal model. Our philosophy is that, if we can estimate this mismatched error in real time, then a robust controller can be designed without the requirement of precise model. From control point of view, we need to estimate in a finite time this unknown uncertainty, and compensate it when designing a feedback controller. Comparing with the existing model-based methods, such as MPC, computedtorque, inverse kinematic, FEM or machine learning technique which have to derive or learn a precise approximated model for the studied soft robot, the proposed method does not need such an assumption.

\section{Problem StATEMENT}

This paper investigates the position control of a 3D printed tripod-type soft robot which is made by silicone. The structure is described in the left picture of Fig. 1. This robot consists of 3 flexible links $A_{i} B_{i}$ for $1 \leq i \leq 3$. On the top, a hole $P$ with diameter $0.5 \mathrm{~cm}$ is designed to hold small object. The objective is to displace the object in the hole. For this, three motors are installed at points $A_{i}$ in order to drive the links $A_{i} B_{i}$ for the purpose of moving the object on the top. The overall dimension of the soft robot is $15 \times 15 \times 9 \mathrm{~cm}$, with $3 \mathrm{D}$ printed hard parts and the molded shell-like silicone piece (the 3 soft links). For the actuators, 3 SG90 servomotors are used, whose rotation angel can be controlled via MegaPi board.

\footnotetext{
${ }^{1}$ https://handsonsoftrobotics.lille.inria.fr
}

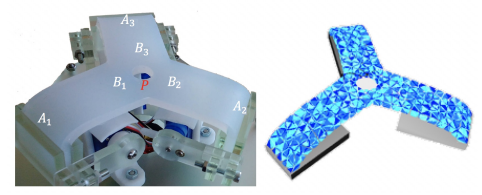

Fig. 1. Left: Tripod-type of 3D printed soft robot; Right: Its' FEM model.

Due to the fact that the links are made by silicone, thus they are flexible. Therefore, the exact kinematic and dynamic models of such a soft robot are quite difficult (or even impossible) to be obtained. One possible solution is to approximate those exact models by using FEM. The basic idea of FEM is to discretize the space of robot by using finite number of fine elements to obtain its dynamical model.

Following the second law of Newton and FEM approach, we can use the following nonlinear model to describe its behavior

$$
M(q) \ddot{q}+D(q, \dot{q}) \dot{q}+K(q) q=H^{T}(q) u
$$

where $q \in \mathbb{R}^{n}$ is the deformation of the nodes of the mesh, and $u \in \mathbb{R}^{m}$ represents the magnitude of the actuators. $M(q)$ is mass matrix which is always invertible, $D(q, \dot{q})$ is damping matrix, and $K(q)$ represents stiffness matrix in order to model the internal forces of the soft robot, which depends on the constitutive law (linear or nonlinear) of the soft material, characterized by the associated Young's modulo and Poison ratio. $H(q)$ represents the force directions (including actuators from the robot itself), and is rectangular operator, usually sparse, as it has only non-zero values at the points where the actuators are applied. For the investigated soft robot, its FEM discretization is displayed in the right picture of Fig. 1, where we can see that the number of nodes is quite huge (1000 nodes in this figure), and it leads to high dimensional state of (1).

In the following, we would like to highlight that the FEMbased controller suffers from at least the following problems:

- The material of the real robot is never homogeneous, and is always spatially variant. This characteristic implies that the method of FEM will never give us a precise model;

- The number of nodes needs to be immense in order to have an acceptable approximation for FEM, and the relation found by FEM depends on the huge matrices $K(q)$ and $H(q)$ in (1). This implies that FEM method is computationally expensive;

- The most important drawback is that: the control developed based on FEM model might not be valid for the real system since FEM is only an approximated model.

In order to avoid the above mentioned inconveniences of FEM, this paper uses a simplified model as the nominal one to describe the dynamics of $P$ with respect to the control input $u$, and the unmatched error will be then represented by the uncertain term. Based on this nominal model with unknown uncertainty, the objective of this paper is then to design robust controller to achieve position control of the object glued on the top of the soft robot. 


\section{MOdELING}

Before presenting the simplified nominal model for this soft robot, a global (inertial) frame needs to be chosen. In this paper, we fix the $x-z$ plane on the base, and choose the center point of the base as the origin $O=(0,0,0)$, and assign one arbitrary motor on the axis $x\left(\overrightarrow{O A}_{1}\right.$ as shown in Fig. 2). Due to the symmetric form of this robot, the $y$-axis is upward and perpendicular to the base.

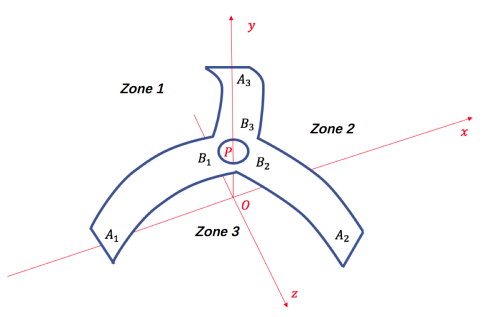

Fig. 2. The chosen global frame fixed on the soft robot.

In this global frame, the coordinate of the top hole of this robot is noted as $P=(x, y, z)^{T} \in \mathbb{R}^{3}$. Since we are interested only in the position control of the top hole, therefore the three mounted motors are enough to achieve this goal.

As we have mentioned in the last section that an exact dynamic model of the point $P$ is quite difficult to be obtained, the following will introduce a simplified nominal linear model with uncertainty to model the dynamics of $P$.

\section{A. Nominal model}

According to the second law of Newton, similarly to (1), we know that the acceleration of an object is proportional to the velocity (the effect of damping and viscous), to the displacement and to the external force. Obviously, the parameters in front of the velocity, the displacement and the inputs might not be constant, like (1). But in practice, we can choose a reasonable constant parameters (via the process of identification, which will be detailed in Section VII) to represent its nominal model. Therefore, the nominal model to describe the dynamics of the top hole for the studied soft robot is assumed to be of the following form:

$$
\ddot{P}=A_{p_{1}} \dot{P}+A_{p_{2}} P+B_{p} u
$$

with $A_{p_{1}} \in \mathbb{R}^{3 \times 3}, A_{p_{2}} \in \mathbb{R}^{3 \times 3}$ and $B_{p} \in \mathbb{R}^{3 \times 3}$. In the workspace of the designed soft tripod robot, the matrix $B_{p}$ should be invertible, this is due to the fact that the position of the top hole $P$ is controllable [17]. The corresponding procedure to identify the values of matrices $A_{p_{1}}, A_{p_{2}}$, and $B_{p}$ will be discussed in Section VII.

With the identified nominal model (2), we can then state that the exact model can be written as

$$
\ddot{P}=A_{p_{1}} P+A_{p_{2}} \dot{P}+B_{p} u+d(t)
$$

where the term $d(t) \in \mathbb{R}^{3}$ represents the unmatched error between the exact model and the identified nominal model (2). We would like to emphasize that, although the nominal model (2) was identified around certain operational positions/trajectories, but the unmatched model parameters in $A_{p_{1}}$,
$A_{p_{2}}$ and $B_{p}$ can be regarded as a resource of the uncertainty $d(t)$. In addition, the unmodeled nonlinearity can be also considered as another source of the uncertainty $d(t)$. In this sense, we can say that the nominal uncertain model (3) enables us to model precisely the movement of the soft robot.

\section{B. Link to FEM}

We can also make a link between the high dimensional FEM model (1) and the low dimensional nominal uncertain model (3). For this, let us define $P=C q$ which represents the position of the top hole of the soft robot. Then, we have $\ddot{P}=C \ddot{q}$ with

$$
\ddot{q}=M^{-1}(q)\left[H^{T}(q) u-D(q, \dot{q}) \dot{q}-K(q) q\right]
$$

Hence we obtain $\ddot{P}=f(q, \dot{q}, \cdots, u)$ which can be written in the nominal uncertain model (3) with

$$
d(t)=f(q, \dot{q}, \cdots, u)-A_{p_{1}} P-A_{p_{2}} \dot{P}-B_{p} u
$$

Such a formulation shows that the constitutive law, described by $K(q)$ in (1), is hidden in $d(t)$. We would like to emphasize that, in the framework of FEM, the constitutive law is normally assumed to be linear (i.e. elastic) for the sake of modeling simplification. Such an assumption is no longer needed when modeling the dynamic behavior of soft robot via the nominal uncertain model (3), since the influence of the constitutive law is now hidden in $d(t)$ which will be estimated and compensated in real time by the closed-loop controller. In other words, such a formulation enables us to treat more complicated constitutive law, such as hyper elastic one.

The objective of our work is then to design a robust controller to solve output regulation/tracking problem of (3) to a desired position $P_{r}$ (not necessary to be constant, which might be time-varying), with the presence of the unknown uncertain term $d(t)$.

\section{ESTIMATION OF UNCERTAINTIES}

In the literature, there exists several approaches to solve the output tracking problem for (3). One solution might be the design of robust controller, such as sliding mode [18], to directly eliminate the influence of bounded uncertainty, since the uncertainty $d(t)$ affects system (3) in a matched way, i.e., the uncertainty $d(t)$ and the control $u(t)$ are in the same line. Theoretically, sliding mode technique can achieve finite-time convergence. However, the gain of such a controller depends on the boundedness of $d(t)$, thus the over-estimation of this boundedness will lead to large gain. In addition, sliding mode controller suffers from chattering phenomenon in practice. Another method to solve the output tracking problem for (3) is based on the idea to estimate the uncertainty $d(t)$, and then compensate it in the closed loop.

In this paper, we will adopt the second solution. To this aim, let us consider the dynamics of $P$ defined in (3). Since $B_{p}$ is invertible, therefore the uncertainty can be easily estimated via $\hat{d}(t)=\ddot{P}-A_{p_{1}} P-A_{p_{2}} \dot{P}$. This formula shows that highorder derivatives of $P$ are required to estimate the unknown uncertainty. Therefore, the following will recall some wellknown differentiators to calculate high-order derivatives. 
The simplest differentiator is the so-called high-gain differentiator [19]. Consider $P$ as the signal for which we want to calculate its high-order derivatives, the high-gain differentiator is of the following form:

$$
\begin{array}{lll}
\dot{\xi}_{1} & =\xi_{2}-k_{1}\left(\xi_{1}-P\right) \\
& \cdots \\
\dot{\xi}_{n-1} & =\xi_{n}-k_{n-1}\left(\xi_{1}-P\right) \\
\dot{\xi}_{n} & =-k_{n}\left(\xi_{1}-P\right)
\end{array}
$$

Define $e_{i}(t)=\xi_{i}(t)-P^{(i-1)}(t)$ where $P^{(i-1)}(t)$ represents the $(i-1)$ th derivative of $P(t)$. Then we can obtain the following dynamics of observation error:

$$
\begin{aligned}
\dot{e}_{1} & =e_{2}-k_{1} e_{1} \\
& \cdots \\
\dot{e}_{n-1} & =e_{n}-k_{2} e_{1} \\
\dot{e}_{n} & =-k_{n} e_{1}-P^{(n)}
\end{aligned}
$$

By assuming that $P^{(n)}$ is bounded (which is always true in practice), it has been proven in [19] that, with the choice of the following gains:

$$
\left[\begin{array}{llll}
k_{1} & k_{2} & \ldots & k_{n}
\end{array}\right]=\left[\begin{array}{llll}
\frac{l_{1}}{\epsilon} & \frac{l_{2}}{\epsilon^{2}} & \ldots & \frac{l_{n}}{\epsilon^{n}}
\end{array}\right]
$$

where $\epsilon$ is a small positive parameter (thus $k_{i}$ is high gain) and $l_{i}$ are positive constants which make the roots of $s^{n}+$ $l_{1} s^{n-1}+\cdots+l_{n-1} s+l_{n}=0$ having negative real parts, then the estimation error $e_{i}$ will decay to $O(\epsilon)$ after a short transient period. Consequently the high-order derivatives of $P$ can be obtained as $\xi_{i} \approx P^{(i-1)}$.

Note that the high-gain differentiator can only provide asymptotic convergence to a small neighborhood of the real derivative. In order to overcome this problem, the so-called HOSM (High-order sliding mode) differentiator and HOMD (Homogeneous finite-time) differentiator are proposed, which are of the following similar form:

$$
\begin{array}{ll}
\dot{\xi}_{1} & =\xi_{2}-k_{1}\left\ulcorner\xi_{1}-P\right\lrcorner^{\alpha} \\
& \cdots \\
\dot{\xi}_{n-1} & =\xi_{n}-k_{n-1}\left\ulcorner\xi_{1}-P\right\lrcorner^{(n-1) \alpha-(n-2)} \\
\dot{\xi}_{n} & =\quad-k_{n}\left\ulcorner\xi_{1}-P\right\lrcorner n \alpha-(n-1)
\end{array}
$$

where $\ulcorner a\lrcorner^{b}=|a|^{b} \operatorname{sign}(a)$ and $k_{i}$ was chosen such that the roots of $s^{n}+k_{1} s^{n-1}+\cdots+k_{n-1} s+k_{n}=0$ having negative real parts. Depending on different choices of $\alpha$, the differentiator (7) is HOMD if $\alpha \in\left(\frac{n-1}{n}, 1\right)$, and it represents a HOSM differentiator if $\alpha=\frac{n-1}{n}$. Following the same procedure stated for high-gain differentiator, the dynamics of observation error becomes:

$$
\begin{aligned}
\dot{e}_{1} & =e_{2}-k_{1}\left\lceil e_{1}\right\rfloor^{\alpha} \\
& \cdots \\
\dot{e}_{n-1} & =e_{n}-k_{n-1}\left\lceil e_{1}\right\rfloor^{(n-1) \alpha-(n-2)} \\
\dot{e}_{n} & =-k_{n}\left\lceil e_{1}\right\rfloor^{n \alpha-(n-1)}-P^{(n)}
\end{aligned}
$$

It has been shown in [20] that the observation error $e_{i}$ will converge to zero in a finite time.

In other words, given the top hole position $P$ of the soft robot, HOSM and HOMD differentiators can precisely calculate $\dot{P}$ and $\ddot{P}$ in a finite time, i.e., $\exists t \geq T_{s}$, such that
$\xi_{1}=P, \xi_{2}=\dot{P}$ and $\xi_{3}=\ddot{P}$. Therefore, the uncertainty term $d(t)$ can be estimated as

$$
\hat{d}(t)=\xi_{3}-A_{p_{1}} \xi_{1}-A_{p_{2}} \xi_{2}
$$

\section{ROBUST CONTROLLER DESIGN}

For the nominal system with uncertainty (3), with uncertainty estimation method (9), we can then design the following robust controller

$u=B_{p}^{-1}\left[-\hat{d}(t)-A_{p_{1}} \xi_{1}-A_{p_{2}} \xi_{2}+\ddot{P}_{r}+K_{p} e+K_{d} \dot{e}\right]$

where $e=P_{r}-\xi_{1}$ and $\dot{e}=\dot{P}_{r}-\xi_{2}$.

Theorem 1. For the robot described by (3), given a desired trajectory $P_{r}(t)$, with the proposed uncertainty estimator (9), there exist $K_{d}$ and $K_{p}$ such that the robust controller (10) can asymptotically track $P_{r}(t)$, i.e., $\lim _{t \rightarrow \infty}\left\|P(t)-P_{r}(t)\right\|=0$.

Proof. For system (3), it is clear that the high-order derivatives of $P$ should be bounded since $P$ represents the position of the top hole of the soft robot. Then, it has been proven in [18] that, for any initial condition $\xi_{i}\left(t_{0}\right)$, there always exists a settling time $T_{s}$ such that for all $t \geq T_{s}$ we have $\xi_{i}(t)=P^{(i-1)}(t)$ for $1 \leq i \leq n$, where $P^{(i-1)}$ represents the $(i-1)$ th derivative of $P$ with respect to time. In other words, after $t \geq T_{s}$, we obtain the exact estimation $\xi_{1}=P, \xi_{2}=\dot{P}$ and $\xi_{3}=\ddot{P}$. According to (9), after $t \geq T_{s}$, we can state that

$$
\hat{d}(t)=\xi_{3}-A_{p_{1}} \xi_{1}-A_{p_{2}} \xi_{2}=\ddot{P}-A_{p_{1}} P-A_{p_{2}} \dot{P}=d(t)
$$

and the corresponding control as

$$
u=B_{p}^{-1}\left[-d(t)-A_{p_{1}} P-A_{p_{2}} \dot{P}+\ddot{P}_{r}+K_{p} e+K_{d} \dot{e}\right]
$$

with $e=P_{r}-P$ and $\dot{e}=\dot{P}_{r}-\dot{P}$. Therefore, substituting the controller (10) back into the nominal uncertain system (3), we can finally get the closed-loop system as follows:

$$
\begin{aligned}
\ddot{P} & =A_{p_{1}} P+A_{p_{2}} \dot{P}+B_{p} u+d(t) \\
& =\ddot{P}_{r}+K_{p} e+K_{d} \dot{e}
\end{aligned}
$$

The above equation can be then rewritten as $\ddot{e}+K_{d} \dot{e}+K_{p} e=$ 0 . Note that this is a 3 -dimensional totally decoupled 2 -order system, therefore we can always find the parameters $K_{d} \in \mathbb{R}^{3}$ and $K_{p} \in \mathbb{R}^{3}$ such that the closed-loop system is stable.

A simple criteria to determine $K_{d}$ and $K_{p}$ is to satisfy the following inequality:

$$
\left[\begin{array}{rc}
0 & I_{3 \times 3} \\
-K_{p} & -K_{d}
\end{array}\right] \prec 0
$$

i.e., negative positive.

By using the Lyapunov function $V=\frac{1}{2} e^{T} e+\frac{1}{2} \dot{e}^{T} \dot{e}$, we can then have

$$
\begin{aligned}
\dot{V} & =e^{T} \dot{e}+\dot{e}^{T} \ddot{e}=e^{T} \dot{e}-\dot{e}^{T} K_{d} \dot{e}-\dot{e}^{T} K_{p} e \\
& =[e, \dot{e}]^{T}\left[\begin{array}{rr}
0 & I_{3 \times 3} \\
-K_{p} & -K_{d}
\end{array}\right]\left[\begin{array}{c}
e \\
\dot{e}
\end{array}\right]<0
\end{aligned}
$$

Therefore, we can conclude that $\lim _{t \rightarrow \infty}\|e(t)\|=0$ which implies that $\lim _{t \rightarrow \infty}\left\|P(t)-P_{r}(t)\right\|=0$. 


\section{EXTERNAL PERTURBATION REJECTION}

Another advantage of the proposed controller is the rejection of external perturbation. To illustrate this characteristic, let us consider the external perturbation added to the body of the soft robot, noted as $F_{d}(t)$, with the following assumption.

Assumption 1. It is assumed that the external perturbation $F_{d}(t)$ is bounded under which there exists a control $u(t)$ such that the $P(t)$ can still reach the desired position $P_{r}$.

This assumption is important for two reasons. Firstly, the external perturbation needs to be bounded which is always true in practice, otherwise the position $P$ will tend to infinity. Secondly, even $F_{d}(t)$ is bounded, there exist certain particular situations such that $P$ cannot, under the additional perturbation $F_{d}(t)$, reach the desired position $P_{r}$ anymore. For example, if $F_{d}(t)$ was opposite (but with the same magnitude) to $u(t)$, then $P$ cannot be controlled in this case. In summary, Assumption 1 is imposed for the purpose of guaranteeing the controllability of $P$ to the desired position $P_{r}$.

Under Assumption 1, the external perturbation $F_{d}(t)$ can also be considered as an additional resource of $d(t)$ in (3). This can be also interpreted by using FEM, whose model is of the following form:

$$
M(q) \ddot{q}+D(q, \dot{q}) \dot{q}+K(q) q=H^{T}(q) u+H_{F}(q, t) F_{d}
$$

where $H_{F}(q, t)$ implies the directions/time where/when the external perturbation $F_{d}(t)$ is applied. Following the same procedure in Section V, we obtain $\ddot{P}=f\left(q, \dot{q}, \cdots, F_{d}, u\right)$ which can be again written as the nominal uncertain model (3) with $d(t)=f\left(q, \dot{q}, \cdots, F_{d}, u\right)-A_{p_{1}} P-A_{p_{2}} \dot{P}-B_{p} u$.

From the above analysis, we can conclude the robustness of the proposed controller via the following result.

Theorem 2. For the soft robot described by (3), given a desired trajectory $P_{r}(t)$, with external perturbation $F_{d}$ satisfying Assumption 1, there exist $K_{d}$ and $K_{p}$ such that the proposed uncertainty estimator (9) and the robust controller (10) can asymptotically track $P_{r}(t)$, i.e., $\lim _{t \rightarrow \infty}\left\|P(t)-P_{r}(t)\right\|=0$.

Proof. It is omitted since it is similar to that of Theorem 2.

\section{EXPERIMENT}

In order to validate the proposed approach, we implement the proposed uncertainty estimator (9) and the robust controller (10) to control the soft robot described in Fig. 1.

\section{A. Experimental setup}

In the experiment, the robot described in Fig. 1 was printed by $3 \mathrm{D}$ printer with silicone. In our test, we are interested in controlling the top hole position of such a silicone robot. Therefore, three markers are glued on the top of the robot (see Fig. 3 (left)). In order to capture in real time the position of the center of these three markers, an OptiTrack system with 4 ultra-red cameras is installed around this robot. These cameras are fixed above the robot and can localize the center position of these three markers with high precision (in millimeter).

We would like to remark two important facts. First, the minimal configuration (i.e., 4 cameras and 3 markers) is

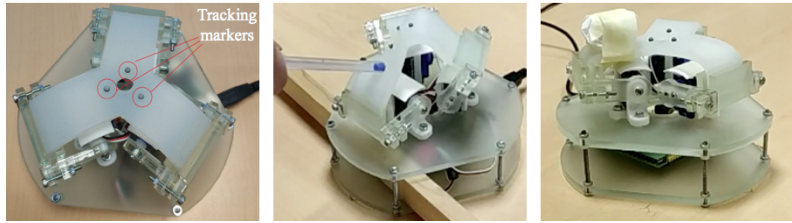

Fig. 3. Experimental setup and external perturbations. Left: Soft robot with 3 markers for OptiTrack tracking; Center: Temporary perturbation was added by touching the soft link with pen; Right: Permanent perturbation was added by gluing an extra material on the soft link.

used during our experiment for easily determining the mass center of all markers, but more markers and cameras can be used for redundancy to account for occlusions which could affect tracking error. Secondly, the proposed controller is independent of the types of sensors we used, if we can measure or estimate the position of the top hole in real time. In other words, different types of sensors (external one such as OptiTrack, or internal one such as air-flow measurement [21]) might be used to measure the position of the top hole, depending on the specific application scenarios.

\section{B. Experimental results}

To show the efficiency of the proposed approach, different experiments are made, respectively to stabilize $P=(x, y, z)$ to a desired position $\left(x_{r}, y_{r}, z_{r}\right)$ (constant or time-varying), without and with external perturbations.

1) Identification of nominal model: In order to identify the values of matrices $A_{p_{1}}, A_{p_{2}}$ and $B_{p}$ in (2), classical identification process has been effectuated for this robot. Precisely, we applied the following inputs

$$
u(t)=\left[\begin{array}{l}
1.5+\sin 0.15 t \\
1+\sin 0.1 t \\
1.3+\sin 0.85 t
\end{array}\right] \times 20, \text { for } 0<t \leq 10
$$

to the three motors, and recorded the corresponding position $P(t)$ via OptiTrack system. Then HOMD differentiator in (7) was used to calculate $\dot{P}(t)$ and $\ddot{P}(t)$. Thus, the nominal model can be written as $\ddot{P}(t)=$ $\left[A_{p_{1}}, A_{p_{2}}, B_{p}\right]\left[\dot{P}^{T}(t), P^{T}(t), u^{T}(t)\right]^{T}$. By using Least-square method, we can then identify the matrices $A_{p_{1}}, A_{p_{2}}$ and $B_{p}$, whose values are:

$$
\begin{aligned}
A_{p_{1}}= & {\left[\begin{array}{rrr}
-0.10 & -0.11 & -0.20 \\
0.35 & -0.21 & -0.20 \\
0.19 & 0.29 & -0.17
\end{array}\right] } \\
A_{p_{2}}= & {\left[\begin{array}{rrr}
-0.13 & 0.12 & -0.67 \\
-0.33 & 0.19 & 0.15 \\
-0.37 & -0.29 & 0.59
\end{array}\right] } \\
B_{p}= & {\left[\begin{array}{rrr}
0.86 & 0.34 & 0.04 \\
-0.25 & 0.73 & -0.32 \\
-0.37 & 0.82 & 0.41
\end{array}\right] }
\end{aligned}
$$

2) Stabilization to a desired position on $y$-axis: In order to show the advantage of the proposed controller, we compared the results with respect to a simple PID controller.

Fig. 4 (left) shows the position errors between $P$ and the desired position by using classical PID controller. It can be 

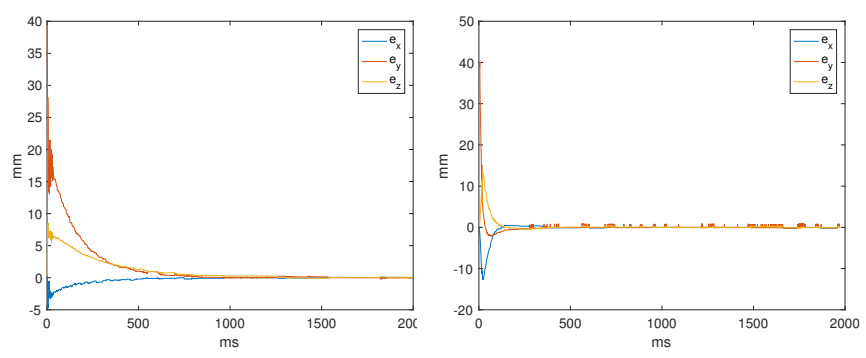

Fig. 4. Stabilization error to a desired position via the PID controller (left) and via controller (10) (right).

seen that PID controller can realize the task around $1000 \mathrm{~ms}$. Fig. 4 (right) depicts the experimental result in the same settings, but by using the proposed controller. It is clear that the proposed controller makes the robot converging to the desired position faster, around $400 \mathrm{~ms}$. This experiment highlights the fast convergence property of the proposed controller with respect to classical PID controller, which is logical since PID controller does not use any information of the model. It is worth noting that the convergence speed can be tuned for PID controller by choosing different parameters. Such a tuning procedure is relatively easy for SISO (Single Input Single Output) system, but generally there does not exist a constructive process to tune those parameters for MIMO (Multi Inputs Multi Outputs) system, which is the case for the studied soft robot: we have 3 coupled inputs, therefore 9 coupled gains need to be carefully tuned for different operating points. For the studied robot, it is clear that different motor has different contribution to move the position of the top hole. Due to this coupling fact, i.e., the modification of one controller will influence the other one, the tuning procedure is time-consuming and will lead to the so-called gain-scheduling control scheme.

3) Stabilization to a desired position in different zones: In order to show the feasibility of the proposed controller, in this experiment we will stabilize the robot to 3 different zones (see Fig. 2 for the definition of zones). Figs. 5-7 show the position errors when stabilizing the robot to a desired constant position located in Zone 1, Zone 2 and Zone 3, respectively. We can see that the proposed controller can achieve the task for all three zones, with a fast convergence speed.

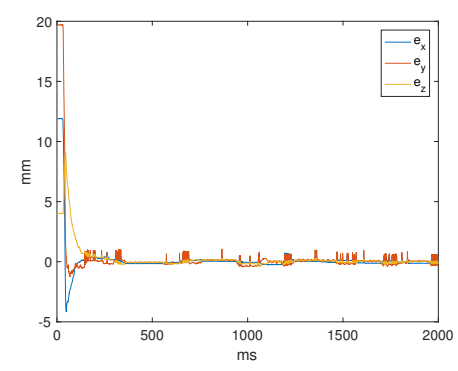

Fig. 5. Stabilization error to a desired position in Zone 1 via controller (10).

4) With external perturbation: In order to show the robustness of the proposed method, two types of perturbations are added to the robot (see the center and right picture in Fig. 3

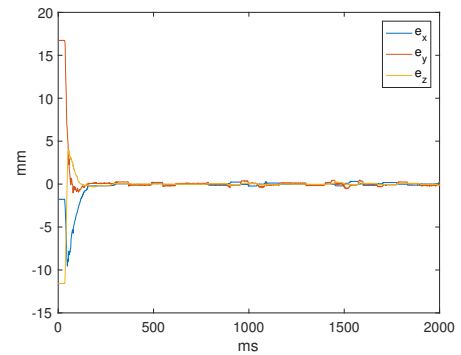

Fig. 6. Stabilization error to a desired position in Zone 2 via controller (10).

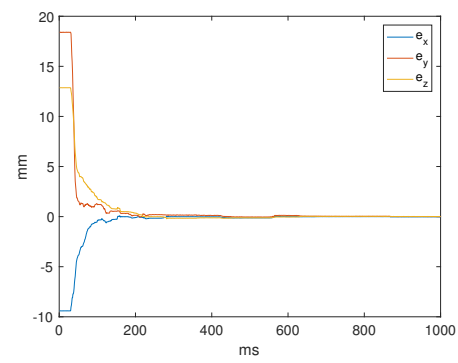

Fig. 7. Stabilization error to a desired position in Zone 3 via controller (10).

). The first one is temporary, where we manually perturb the robot with a pen and then release it. Fig. 8 (left) shows the experimental results. We can see that the proposed controller can keep the robot on the desired position when such an external temporary perturbation is presented and vanished.

The second type of perturbation is permanent. We manually glue an extra material on the body of soft robot, for the purpose of mimicking an external perturbation which is present permanently. As shown in Fig. 8 (right), the proposed approach can reject this perturbation, even it is permanent.

From those experimental results, it is clear that the proposed robust controller can successfully drive the point of interest (the center of three markers glued on the top of robot) to a desired constant position in different zones, and it is robust with respect to external perturbation.

5) Trajectory tracking: For the purpose of tracking a timevarying trajectory, we define the following references

$$
P_{r}(t)=\left[\begin{array}{l}
x_{r}(t) \\
y_{r}(t) \\
z_{r}(t)
\end{array}\right]=\left[\begin{array}{c}
10 \sin 0.5 t-10 \\
10 \cos 0.6 t+20 \\
10 \sin 0.8 t+10
\end{array}\right]
$$
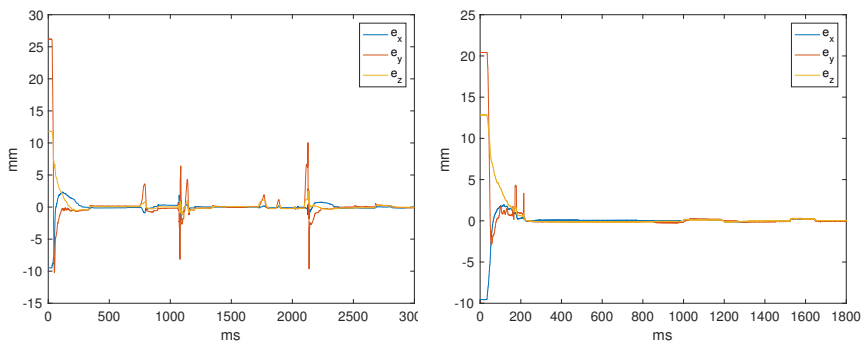

Fig. 8. Robustness with respect to an external temporary perturbation (left) and an external permanent perturbation (right). 
Moreover, in order to highlight the efficiency of the uncertainty compensation, two tests are made, respectively with the estimation and compensation of the uncertainty and without it. The relative results are shown in Fig. 9, where we can see that, the controller (10) without the compensation of the uncertainty $\hat{d}(t)$ cannot achieve the trajectory tracking task. Compared to the trajectory reference, it has a bounded but oscillatory tendency. This phenomenon can be interpreted as follows: the uncompensated term $d(t)$, which is used to catch the unmatched modeling error between the linear nominal model and the real one, might be nonlinear in some neighborhoods of the reference, therefore the robot cannot converge to the reference by the controller (10) without the uncertainty compensation. Conversely, when integrating the compensation of this uncertainty, the proposed controller can successfully track the defined time-varying trajectory. This experiment clearly shows that the estimation and compensation of the uncertainty term $d(t)$ plays an important role of the proposed controller.

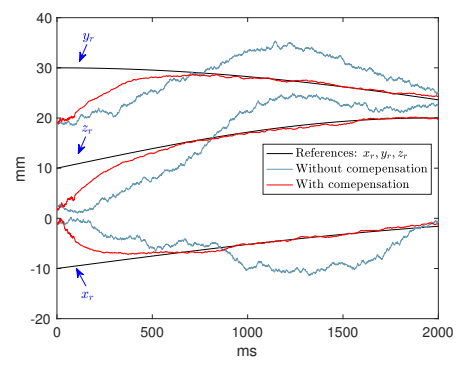

Fig. 9. Performance comparisons for the proposed control without and with the uncertainty compensation for tracking time-varying references.

\section{CONCLUSION}

In this paper, we proposed to use a simplified linear nominal model with uncertainty to describe the dynamics of soft robots. By analyzing the disadvantage of classical FEM approach to model soft robot, we showed the feasibility of the proposed approach by linking the FEM model to the proposed one. Then, the problem of position control of soft robot is converted to investigate the estimation of uncertainty and the design of robust controller by compensating the estimated uncertainty. In this paper, different types of uncertainty estimators have been discussed. Also, the scenario with external perturbation has been analyzed for the purpose of highlighting the robustness of the proposed controller. Finally, different types of experiments have been carried out, including the stabilization to a constant or time-varying desired position in different zones, and the case with external perturbation. The experimental results show that the proposed approach is efficient, and robust to achieve position control of the investigated soft robot.

\section{REFERENCES}

[1] D. Trivedi, C. D. Rahn, W. M. Kier, and I. D. Walker, "Soft robotics: Biological inspiration, state of the art, and future research," Appl. Bionics Biomechanics, vol. 5, no. 3, pp. 99-117, 2008.
[2] S. Wittmeier, C. Alessandro, N. Bascarevic, K. Dalamagkidis, D. Devereux, A. Diamond, M. Jäntsch, K. Jovanovic, R. Knight, H. G. Marques, P. Milosavljevic, B. Mitra, B. Svetozarevic, V. Potkonjak, R. Pfeifer, A. Knoll, and O. Holland, "Toward anthropomimetic robotics: Development, simulation, and control of a musculoskeletal torso," Artificial Life, vol. 19, no. 1, pp. 171-193, 2013.

[3] C. M. Best, M. T. Gillespie, P. Hyatt, L. Rupert, V. Sherrod, and M. D. Killpack, "A new soft robot control method: Using model predictive control for a pneumatically actuated humanoid," IEEE Robotics Automation Magazine, vol. 23, no. 3, pp. 75-84, Sept 2016.

[4] C. Laschi and M. Cianchetti, "Soft robotics: new perspectives for robot bodyware and control," Frontiers in bioengineering and biotechnology, vol. 2, p. 3, 2014.

[5] G. S. Chirikjian and J. W. Burdick, "A modal approach to hyperredundant manipulator kinematics," IEEE Transactions on Robotics and Automation, vol. 10, no. 3, pp. 343-354, 1994.

[6] F. Boyer, M. Porez, and W. Khalil, "Macro-continuous computed torque algorithm for a three-dimensional eel-like robot," IEEE Transactions on Robotics, vol. 22, no. 4, pp. 763-775, 2006.

[7] D. B. Camarillo, C. R. Carlson, and J. K. Salisbury, "Configuration tracking for continuum manipulators with coupled tendon drive," IEEE Transactions on Robotics, vol. 25, no. 4, pp. 798-808, Aug 2009.

[8] C. Della Santina, R. K. Katzschmann, A. Bicchi, and D. Rus, "Dynamic control of soft robots interacting with the environment," 2018.

[9] J. B. M. Siddharth Sanan and C. G. Atkeson, "Robots with inflatable links," in The 2009 IEEE/RSJ International Conference on Inte lligent Robots and Systems October 11-15, 2009 St. Louis, USA. IEEE, 2009.

[10] B. A. Jones, R. L. Gray, and K. Turlapati, "Three dimensional statics for continuum robotics," in 2009 IEEE/RSJ International Conference on Intelligent Robots and Systems, Oct 2009, pp. 2659-2664.

[11] F. Renda, M. Cianchetti, M. Giorelli, A. Arienti, and C. Laschi, "A $3 \mathrm{~d}$ steady-state model of a tendon-driven continuum soft manipulator inspired by the octopus arm," Bioinspiration \& biomimetics, vol. 7, no. 2, p. 025006, 2012.

[12] Z. Zhang, J. Dequidt, and C. Duriez, "Vision-Based Sensing of External Forces Acting on Soft Robots Using Finite Element Method," IEEE Robotics and Automation Letters, vol. 3, no. 3, pp. 1529 - 1536, Feb. 2018.

[13] D. Braganza, D. M. Dawson, I. D. Walker, and N. Nath, "A neural network controller for continuum robots," IEEE transactions on robotics, vol. 23, no. 6, pp. 1270-1277, 2007.

[14] A. D. Marchese, R. Tedrake, and D. Rus, "Dynamics and trajectory optimization for a soft spatial fluidic elastomer manipulator," The International Journal of Robotics Research, vol. 35, no. 8, pp. 10001019, 2016.

[15] T. G. Thuruthel, E. Falotico, F. Renda, and C. Laschi, "Learning dynamic models for open loop predictive control of soft robotic manipulators," Bioinspiration \& biomimetics, vol. 12, no. 6, p. 066003, 2017.

[16] G. Zheng, Y. Zhou, and M. Ju, "Robust control of a silicone soft robot using neural networks," ISA Transactions, accepted, 2019.

[17] G. Zheng, O. Goury, M. Thieffry, A. Kruszewski, and C. Duriez, "Controllability pre-verification of silicon soft robots based on finiteelement method," in Robotics and Automation (ICRA), 2019 IEEE International Conference on.

[18] A. Levant, "Sliding order and sliding accuracy in sliding mode control," International journal of control, vol. 58, no. 6, pp. 1247-1263, 1993.

[19] H. K. Khalil, "High-gain observers in nonlinear feedback control," in 2008 International Conference on Control, Automation and Systems. IEEE, 2008, pp. xlvii-lvii.

[20] Y. Wang, G. Zheng, D. Efimov, and W. Perruquetti, "Differentiator application in altitude control for an indoor blimp robot," International Journal of Control, pp. 1-10, 2018.

[21] S. E. Navarro, O. Goury, G. Zheng, T. M. Bieze, and C. Duriez, "Modeling novel soft mechanosensors based on air-flow measurements," IEEE Robotics and Automation Letters, vol. 4, no. 4, pp. 4338-4345, 2019. 